\title{
Erratum
}

\section{Theory of the Photon-Drag Effect in $p$-Type Germanium with a Parabolic and Anisotropic Band-Structure Approximation}

\author{
T. Grave, H. Wurz, W. Schneider, and K. Hübner \\ Institut für Angewandte Physik II, Universität, D-6900 Heidelberg, Fed. Rep. Germany
}

Appl. Phys. 15, 433-438 (1978)

Unfortunately, the integration over the solid angle in (2) was not carried out correctly. Of course, (2) should read

$$
\begin{aligned}
\mathbf{j}(s)= & \int_{\varphi=0}^{2 \pi} \int_{\vartheta=0}^{\pi} e \Delta p_{h}(s) \mathbf{v}_{h}(\vartheta, \varphi) \sin \vartheta d \vartheta d \varphi \\
& +\int_{\varphi=0}^{2 \pi} \int_{\vartheta=0}^{\pi} e \Delta p_{l}(s) \mathbf{v}_{l}(\vartheta, \varphi) \sin \vartheta d \vartheta d \varphi .
\end{aligned}
$$

The integrals in (4), (7), (9), and (10) must be corrected correspondingly, and (6) must be replaced by

$\alpha_{k}=\alpha_{h} / 4 \pi$.

This new expression for $\alpha_{k}$ implies that $\pi^{2}$ in the denominator of (7), (11), (25), (26), (28), (29), and (31) must be replaced by $\pi$. Our numerical calculations were repeated with the given modifications yielding $D_{L}$ to be independent of the crystallographic direction, and $D_{T[111]}$ vanished. These results are due to our introduction of a transition probability independent of polarisation which means a reduction of the fourthrank photon-drag tensor to a tensor of the second rank. It can be shown that properties described by second-rank tensors are completely isotropic in crystals with cubic symmetry [2].
The new numerical result (replacing the one shown in Fig. 2) is $D_{L}=-0.55+0.55 \tau_{h} / \tau_{l}$. For $p>10^{15} \mathrm{~cm}^{-3}$, Hattori et al. [3] measured the tensor components $S=1.3 \times 10^{-7} \mathrm{~cm} / \mathrm{A}$ and $(P+2 S) / 3=1.7 \times 10^{-7} \mathrm{~cm} / \mathrm{A}$ corresponding to $D_{L_{[100]}}=1.1$ and $D_{L[111]}=1.4$, respectively. Measurement by $\mathrm{N}$. Wenzel at our institute resulted in $S \approx 3.3 \times 10^{-7} \mathrm{~cm} / \mathrm{A}$ and $(P+2 S) / 3$ $\approx 4.2 \times 10^{-7} \mathrm{~cm} / \mathrm{A}$ for $p=4 \times 10^{14} \ldots 2 \times 10^{16} \mathrm{~cm}^{-3}$. This means that $D_{L[100]} \approx 2.6$ and $D_{L[111]} \approx 3.3$. Acoustical and optical scattering rates [4] at the energies of the final and initial states of the transition lead to $\tau_{l} \approx 0.1 \mathrm{ps}$ and $\tau_{h} \approx 0.6 \mathrm{ps}$. From the measurements of Hattori et al. $\tau_{h} \approx 0.4$ ps can be calculated, and our own experiments resulted in $\tau_{h}=0.7 \ldots 0.9 \mathrm{ps}$, if $\tau_{l}=0.1 \mathrm{ps}$ is assumed.

\section{References}

1. A.C.Walker, D.R. Tilley: J. Phys. C4, L376-378 (1971)

2. J.F.Nye: Physical Properties of Crystals (Oxford University Press, Oxford 1957) p. 23

3. H.Hattori, M.Umeno, T.Jimbo, O.Fujitani, S.Miki : J. Phys. Soc. Jap. 35, 826-831 (1973)

4. D.M. Brown, R.Bray: Phys. Rev. 127, 1593-1602 (1962) 